\title{
Accounting for Some Aspects of Dark Matter and Dark Energy via Noncommutative Geometry
}

\author{
Peter K. F. Kuhfittig \\ Department of Mathematics, Milwaukee School of Engineering, Milwaukee, WI, USA \\ Email:kuhfitti@msoe.edu
}

How to cite this paper: Kuhfittig, P.K.F. (2017) Accounting for Some Aspects of Dark Matter and Dark Energy via Noncommutative Geometry. Journal of Modern Physics, 8, 323-329.

https://doi.org/10.4236/jmp.2017.83021

Received: October 25, 2016

Accepted: February 25, 2017

Published: February 28, 2017

Copyright $\odot 2017$ by author and Scientific Research Publishing Inc. This work is licensed under the Creative Commons Attribution International License (CC BY 4.0).

http://creativecommons.org/licenses/by/4.0/

\begin{abstract}
The purpose of this paper is to seek a connection between noncommutative geometry, an offshoot of string theory, and certain aspects of dark matter and dark energy. The former case is based on a simple mathematical argument showing that the main manifestation of dark matter in connection with flat galactic rotation curves is also a consequence of noncommutative geometry. The latter case requires an examination of the local effect of noncommutative geometry and the subsequent extension to the global phenomenon of an accelerating Universe.
\end{abstract}

\section{Keywords}

Noncommutative Geometry, Dark Matter, Dark Energy

\section{Introduction}

While it is generally assumed that dark matter is needed to account for galactic rotation curves in the outer region of galaxies, it has already been observed that a noncommutative-geometry background can accomplish this goal equally well [1] [2]. In the first part of this paper, we seek a mathematical explanation for this outcome: both dark matter and noncommutative geometry predict that the mass inside a sphere of radius $r$ increases linearly with $r$ in the outward radial direction. It follows that dark matter is not needed to account for flat galactic rotation curves.

The second part of this paper makes an analogous connection between noncommutative geometry and dark energy. An analysis of the local effect of noncommutative geometry suggests an extension thereof to the global phenomenon of an accelerating Universe. 
The main conclusion is that string theory in the form of a noncommutativegeometry background can account for certain aspects of both dark matter and dark energy.

\section{Noncommutative Geometry}

Suppose we start with the general metric of a static spherically symmetric line element, using units in which $c=G=1$ :

$$
\mathrm{d} s^{2}=-e^{2 \Phi(r)} \mathrm{d} t^{2}+\frac{\mathrm{d} r^{2}}{1-\frac{2 m(r)}{r}}+r^{2}\left(\mathrm{~d} \theta^{2}+\sin ^{2} \theta \mathrm{d} \phi^{2}\right) ;
$$

here $m(r)$ is the effective mass inside a sphere of radius $r$ with $m(0)=0$. We also require that $\lim _{r \rightarrow \infty} m(r) / r=0$.

Because of the spherical symmetry, the only nonzero components of the stress-energy tensor are $T_{t}^{t}=-\rho(r)$, the energy density, $T_{r}^{r}=p_{r}(r)$, the radial pressure, and $T_{\theta}^{\theta}=T_{\phi}^{\phi}=p_{t}(r)$, the lateral pressure. The Einstein field equations can be written in the following form:

$$
\begin{gathered}
\rho(r)=\frac{2 m^{\prime}}{8 \pi r^{2}}, \\
p_{r}(r)=\frac{1}{8 \pi}\left[-\frac{2 m}{r^{3}}+\frac{2 \Phi^{\prime}}{r}\left(1-\frac{2 m}{r}\right)\right],
\end{gathered}
$$

and

$$
p_{t}(r)=\frac{1}{8 \pi}\left(1-\frac{2 m}{r}\right)\left[\Phi^{\prime \prime}-\frac{2 m^{\prime} r-2 m}{2 r(r-2 m)} \Phi^{\prime}+\left(\Phi^{\prime}\right)^{2}+\frac{\Phi^{\prime}}{r}-\frac{2 m^{\prime} r-2 m}{2 r^{2}(r-2 m)}\right] .
$$

The conservation law $T_{\beta ; \alpha}^{\alpha}=0$ implies that

$$
p_{r}^{\prime}+\Phi^{\prime} \rho+\Phi^{\prime} p_{r}+\frac{2 p_{r}}{r}-\frac{2 p_{t}}{r}=0
$$

As a result, only Equations (2) and (3) are actually needed, an observation that will be taken advantage of later.

Next, we take a brief look at noncommutative geometry, an area that is based on the following outcome of string theory: coordinates may become noncommuting operators on a $D$-brane [3] [4]. Here the commutator is $\left[x^{\mu}, x^{v}\right]=i \theta^{\mu v}$, where $\theta^{\mu v}$ is an antisymmetric matrix. The main idea, discussed in Refs. [5] [6], is that noncommutativity replaces point-like structures by smeared objects. (The aim is to eliminate the divergences that normally occur in general relativity). A natural way to accomplish the smearing effect is to use a Gaussian distribution of minimal length $\sqrt{\beta}$ rather than the Dirac delta function [7] [8]. An equivalent, but simpler, way is to assume that the energy density of the static and spherically symmetric and particle-like gravitational source has the form [9] [10]

$$
\rho(r)=\frac{M \sqrt{\beta}}{\pi^{2}\left(r^{2}+\beta\right)^{2}} .
$$

The point is that the mass $M$ of the particle is diffused throughout the region of linear dimension $\sqrt{\beta}$ due to the uncertainty. 
To make use of Equation (6), one can keep the standard form of the Einstein field equations in the sense that the Einstein tensor retains its original form but the stress-energy tensor is modified [7]. It follows that the length scale need not be restricted to the Planck scale. It is further noted in Ref. [7] that noncommutative geometry is an intrinsic property of spacetime and does not depend on any particular feature such as curvature.

The gravitational source in Equation (6) results in a smeared mass. As in Refs. [5] [6], the Schwarzschild solution of the Einstein field equations associated with the smeared source leads to the line element

$$
\mathrm{d} s^{2}=-\left(1-\frac{2 M_{\beta}(r)}{r}\right) \mathrm{d} t^{2}+\left(1-\frac{2 M_{\beta}(r)}{r}\right)^{-1} \mathrm{~d} r^{2}+r^{2}\left(d \theta^{2}+\sin ^{2} \theta \mathrm{d} \phi^{2}\right) .
$$

Here the smeared mass is found to be

$$
M_{\beta}(r)=\int_{0}^{r} 4 \pi\left(r^{\prime}\right)^{2} \rho\left(r^{\prime}\right) \mathrm{d} r^{\prime}=\frac{2 M}{\pi}\left(\tan ^{-1} \frac{r}{\sqrt{\beta}}-\frac{r \sqrt{\beta}}{r^{2}+\beta}\right) .
$$

Since $\lim _{r \rightarrow 0} M_{\beta}(r) / r=0$, there is no singularity at $r=0$.

Due to the smearing, the mass of the particle depends on $\beta$, as well as on $r$. As in the case of the Gaussian model, the mass of the particle is zero at the center and rapidly increases to $M$. As a result, from a distance the smearing is no longer observed and we get an ordinary particle:

$$
\lim _{\beta \rightarrow 0} M_{\beta}(r)=M \text {. }
$$

So the modified Schwarzschild solution becomes an ordinary Schwarzschild solution in the limit.

\section{The Dark-Matter Hypothesis}

The existence of dark matter was already hypothesized in the 1930's by Zwicky and others. The implications thereof were not recognized until the 1970's when it was observed that galaxies exhibit flat rotation curves (constant velocities) sufficiently far from the galactic center [11]. This observation indicates that the matter in the galaxy increases linearly in the outward radial direction.

To recall the reason for this, suppose $m_{1}$ is the mass of a star, $v$ its constant velocity, and $m_{2}$ the mass of everything else. Now multiplying $m_{1}$ by the centripetal acceleration yields

$$
m_{1} \frac{v^{2}}{r}=m_{1} m_{2} \frac{G}{r^{2}},
$$

where $G$ is Newton's gravitational constant. Using geometrized units $(G=c=1)$, we obtain the linear form

$$
m_{2}=r v^{2}
$$

as asserted. Equation (10) essentially characterizes the dark-matter hypothesis.

Consider next a thin spherical shell of radius $r=r_{0}$. So instead of a smeared object located at the origin, we now have a smeared spherical surface. We consider the smearing in the outward radial direction only, since that is the 
analogue of the smeared particle at the origin. The energy density in Equation (6) must therefore be replaced by

$$
\rho(r)=\frac{M \sqrt{\beta}}{\pi^{2}\left[\left(r-r_{0}\right)^{2}+\beta\right]^{2}},
$$

which is simply a translation in the $r$-direction. Then the smeared mass of the shell becomes

$$
m_{\beta}(r)=\frac{2 M}{\pi}\left[\tan ^{-1} \frac{r-r_{0}}{\sqrt{\beta}}-\frac{\left(r-r_{0}\right) \sqrt{\beta}}{\left(r-r_{0}\right)^{2}+\beta}\right] .
$$

Observe that, analogously,

$$
\lim _{\beta \rightarrow 0} m_{\beta}(r)=M .
$$

So the mass of the shell is zero at $r=r_{0}$ and rapidly rises to $M$.

Since we are moving in the outward radial direction, we can replace "mass of the shell" by "mass per unit length in the $r$-direction", denoted by $m_{\beta}(r)$ in Equation (12). Alternatively, if $M_{T}(r)$ is the total mass, then the mass of the shell of thickness $d r$ becomes the differential

$$
\mathrm{d} M_{T}(r)=\frac{\mathrm{d} M_{T}(r)}{\mathrm{d} r} \mathrm{~d} r=m_{\beta}(r) \mathrm{d} r .
$$

Either way, $m_{\beta}(r)$ and $M$ in Equation (12) are dimensionless in our geometrized units. The total mass is therefore given by

$$
\begin{aligned}
M_{T}(r) & =\int_{0}^{r} m_{\beta}\left(r^{\prime}\right) \mathrm{d} r^{\prime}=\frac{2 M}{\pi}\left[\left(r-r_{0}\right) \tan ^{-1} \frac{r-r_{0}}{\sqrt{\beta}}-\sqrt{\beta} \ln \left[\left(r-r_{0}\right)^{2}+\beta\right]\right] \\
& =\frac{2 M}{\pi}\left(r-r_{0}\right)\left[\tan ^{-1} \frac{r-r_{0}}{\sqrt{\beta}}-\sqrt{\beta} \frac{\ln \left[\left(r-r_{0}\right)^{2}+\beta\right]}{r-r_{0}}\right] .
\end{aligned}
$$

(So $M_{T}(r)$ has units of length). For the expression inside the brackets, we have

$$
\lim _{r \rightarrow \infty}\left[\tan ^{-1} \frac{r-r_{0}}{\sqrt{\beta}}-\sqrt{\beta} \frac{\ln \left[\left(r-r_{0}\right)^{2}+\beta\right]}{r-r_{0}}\right]=\frac{\pi}{2}-0 .
$$

It follows that $M_{T}(r)$ has the linear form

$$
M\left(r-r_{0}\right),
$$

in agreement with Equation (10). Not only does this provide an alternative to the dark-matter hypothesis, the geometric interpretation of the gravitational pull due to dark matter is very much in the spirit of Einstein's theory, which replaces the concept of gravitational force by the geometric concept of curvature.

Remark. It is important to note that we are examining only one aspect of dark matter, accounting for galactic rotation curves. Evidence for dark matter also comes from other sources, such as the need to explain the CMB temperature anisotropy, which is beyond the scope of this study. On the other hand, it is 
shown in Ref. [12] that dark matter also emerges from noncommutative geometry in a more general cosmological setting.

\section{Dark Energy}

A major discovery in the late 1990's was that our Universe is undergoing an accelerated expansion [13] [14], i.e., $\ddot{a}>0$ in the Friedmann equation

$$
\frac{\ddot{a}(t)}{a(t)}=-\frac{4 \pi}{3}(\rho+3 p) \text {. }
$$

Here $p=p_{r}=p_{t}$ since in a cosmological setting we are dealing with a homogeneous distribution of matter. The acceleration is caused by a negative pressure dark energy. In particular, if the equation of state is $p=\omega \rho$, then a value of $\omega<-1 / 3$ is required for an accelerated expansion (Current data favor $\omega=-1$, which is equivalent to assuming Einstein's cosmological constant [15]).

To make use of

$$
\rho(r)=\frac{M \sqrt{\beta}}{\pi^{2}\left(r^{2}+\beta\right)^{2}}
$$

in the cosmological model (15), we need to recall that our Universe is a 3-sphere, having neither a center nor an edge. So any point can be chosen for the origin of the above $\rho(r)$. Moreover, the scale factor $a(t)$ in the FLRW model

$$
\mathrm{d} s^{2}=-\mathrm{d} t^{2}+a(t)\left[\frac{\mathrm{d} r^{2}}{1-K r^{2}}+r^{2}\left(\mathrm{~d} \theta^{2}+\sin ^{2} \theta \mathrm{d} \phi^{2}\right)\right]
$$

refers to Equation (15). Equation (16) now suggests that $\Phi(r) \equiv 0$ for the function $\Phi(r)$ in Equation (1). So for an arbitrarily chosen particle, the line element becomes

$$
\mathrm{d} s^{2}=-\mathrm{d} t^{2}+\frac{\mathrm{d} r^{2}}{1-\frac{2 m(r)}{r}}+r^{2}\left(\mathrm{~d} \theta^{2}+\sin ^{2} \theta \mathrm{d} \phi^{2}\right)
$$

where $m(r)=M_{\beta}(r)$ in Equation (8).

Recalling that $\Phi \equiv 0$, Equation (3) now yields

$$
\rho+3 p=\frac{M \sqrt{\beta}}{\pi^{2}\left(r^{2}+\beta\right)^{2}}+\frac{3}{8 \pi}\left(-\frac{1}{r^{3}}\right) \frac{4 M}{\pi}\left(\tan ^{-1} \frac{r}{\sqrt{\beta}}-\frac{r \sqrt{\beta}}{r^{2}+\beta}\right)
$$

but only near the origin.

Before trying to generalize this result to a cosmological setting, let us examine $\rho+3 p$ in the neighborhood of the origin by letting $r=a \sqrt{\beta}, a>0$. Equation (18) now yields

$$
-\frac{4 \pi}{3}(\rho+3 p)=-\frac{4 \pi}{3} \frac{M}{\pi^{2}} \frac{1}{\beta^{3 / 2}}\left[\frac{1}{\left(a^{2}+1\right)^{2}}-\frac{3}{2 a^{3}}\left(\tan ^{-1} a-\frac{a}{a^{2}+1}\right)\right] .
$$

The result can best be seen qualitatively by plotting $\rho+3 p$ against $a$, as shown in Figure 1. So $\rho+3 p$ is zero at the origin, then becomes negative, 


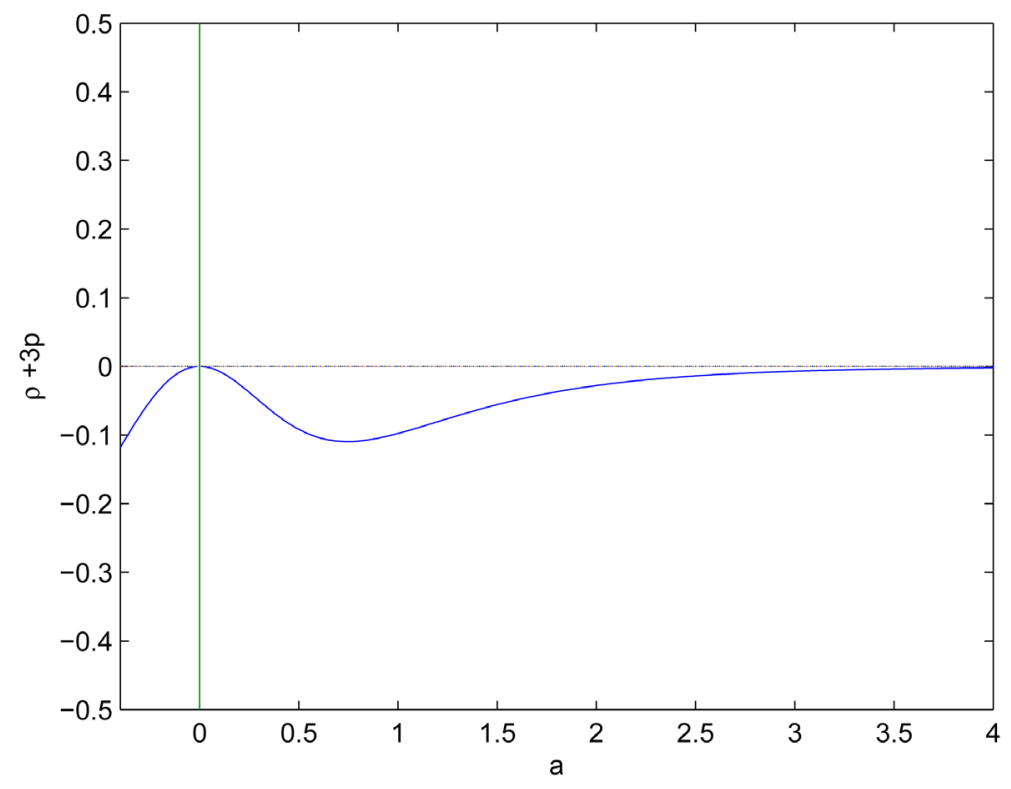

Figure 1. $\rho+3 p$ is plotted against $a$.

before approaching zero asymptotically. The asymptotic behavior is to be expected since from a distance, the smearing is no longer apparent.

We can now assert that

$$
-\frac{4 \pi}{3}(\rho+3 p)>0
$$

in the neighborhood of every particle. Moreover, we are now in a vacuum, which is teeming with virtual particles. These particles are extremely short lived, but given that all particles have a finite lifetime, it is generally assumed that there is no absolute distinction between virtual and ordinary particles. Inequality (20) would therefore hold in the vicinity of every point. The cumulative effect would therefore be an accelerated expansion on a cosmological scale.

Inequality (20) is consistent with the discussion in Ref. [7], which asserts that the collapse of a smeared particle to a point mass is prevented by a kind of "quantum pressure", an outward push induced by noncommuting coordinate quantum fluctuations.

\section{Conclusions}

This paper discusses certain manifestations of string theory in the form of noncommutative geometry. The first part of this paper examines a particular aspect of dark matter, accounting for flat galactic rotation curves. It is shown that a noncommutative-geometry background agrees with the dark-matter assumption, whose basic manifestation is the linearly increasing mass in the outward radial direction. Connections to other aspects of dark matter, such as structure formation, are thereby left open. However, according to Ref. [12], dark matter does emerge from noncommutative geometry in a cosmological setting; the same is true for dark energy.

The second part of this paper discusses a particular aspect of dark energy by 
examining the local effect of the smearing that characterizes noncommutative geometry. Since the Universe is a 3-sphere, any point can serve as the origin for $\rho(r)$. As discussed at the end of Sec. 4 , in a vacuum, the existence of virtual particles everywhere then implies that $(-4 \pi / 3)(\rho+3 p)>0$ in the neighborhood of the origin and hence of every point. The cumulative effect is an acceleration on a cosmological scale. So if the virtual particles are indeed the cause of the acceleration, then it is appropriate to say that dark energy can be viewed as vacuum energy.

Concluding comment: accounting for both dark matter and dark energy may be considered a promissing step toward obtaining empirical evidence for string theory, given that noncommutative geometry is an offshoot thereof.

\section{References}

[1] Rahaman, F., Kuhfittig, P.K.F., Chakraborty, K., Usmani, A.A. and Ray, S. (2012) General Relativity and Gravitation, 44, 905-916. https://doi.org/10.1007/s10714-011-1320-5

[2] Kuhfittig, P.K.F. and Gladney, V. (2014) Journal of Modern Physics, 5, 1931-1937. https://doi.org/10.4236/jmp.2014.517187

[3] Witten, E. (1996) Nuclear Physics B, 460, 335-350. https://doi.org/10.1016/0550-3213(95)00610-9

[4] Seiberg, N. and Witten, E. (1999) Journal of High Energy Physics, 9909, Article ID: 032.

[5] Smailagic, A. and Spallucci, E. (2003) Journal of Physics A, 36, L-467-L-471. https://doi.org/10.1088/0305-4470/36/33/101

[6] Smailagic, A. and Spallucci, E. (2003) Journal of Physics A, 36, L-517-L-521. https://doi.org/10.1088/0305-4470/36/39/103

[7] Nicolini, P., Smailagic, A. and Spallucci, E. (2006) Physics Letters B, 632, 547-551. https://doi.org/10.1016/j.physletb.2005.11.004

[8] Kuhfittig, P.K.F. (2013) International Journal of Pure and Applied Mathematics, 89, 401-408. https://doi.org/10.12732/ijpam.v89i3.11

[9] Nozari, K. and Mehdipour, S.H. (2008) Classical and Quantum Gravity, 25, Article ID: 175015. https://doi.org/10.1088/0264-9381/25/17/175015

[10] Kuhfittig, P.K.F. (2015) International Journal of Modern Physics D, 24, Article ID: 1550023. https://doi.org/10.1142/S0218271815500236

[11] Rubin, V., Thonnard, N. and Ford, W.K. (1980) Astrophysical Journal, 238, 471487. https://doi.org/10.1086/158003

[12] Chamsedding, A.H., Connes, A. and Mukhanov, V. (2015) Physical Review Letters, 114, Article ID: 091302.

[13] Riess, A.G., et al. (1998) Astronomical Journal, 116, 1009-1038. https://doi.org/10.1086/300499

[14] Perlmutter, S.J., et al. (1999) Astrophysical Journal, 517, 565-586. https://doi.org/10.1086/307221

[15] Carmeli, M. (2001) Accelerating Universe, Cosmological Constant and Dark Energy. arXiv: asro-ph/0111259. 
Submit or recommend next manuscript to SCIRP and we will provide best service for you:

Accepting pre-submission inquiries through Email, Facebook, LinkedIn, Twitter, etc. A wide selection of journals (inclusive of 9 subjects, more than 200 journals)

Providing 24-hour high-quality service

User-friendly online submission system

Fair and swift peer-review system

Efficient typesetting and proofreading procedure

Display of the result of downloads and visits, as well as the number of cited articles Maximum dissemination of your research work

Submit your manuscript at: http://papersubmission.scirp.org/

Or contact jmp@scirp.org 